"This is an Accepted Manuscript of an article published by Ind. \& Eng. Chem. Res. (I \& EC R), of American Chemical Society (ACS), vol 59 (issue no 16), pgs 74267437, accepted on March 20, 2020."

\title{
Designing Optimal Terpolymers for Enhanced Oil Recovery (Polymer Flooding)
}

\author{
Alison J. Scott and Alexander Penlidis* \\ Institute for Polymer Research, Department of Chemical Engineering, University of Waterloo, \\ 200 University Avenue West, Waterloo, Ontario, N2L 3G1, Canada; \\ E-mail: penlidis@uwaterloo.ca
}

KEYWORDS: design of experiments; enhanced oil recovery; polymer flooding; reactivity ratio estimation; terpolymerization; rheology; viscoelastic behaviour.

ABSTRACT: Terpolymers of 2-acrylamido-2-methylpropane sulfonic acid (AMPS), acrylamide (AAm) and acrylic acid (AAc) have significant potential to be used in polymer flooding for enhanced oil recovery. Our earlier work has explored the relationship between terpolymerization recipes and polymer properties. With a good understanding of these relationships, two optimal terpolymers have been designed for the application. Relationships between the pre-polymerization 
formulation, product terpolymer composition, polymer chain microstructure, thermal stability, and viscoelastic properties are explored. The synthesis and characterization stages lay the groundwork and establish the viability of these materials for future polymer flooding investigations.

\section{Introduction}

In general, the most widely used synthetic polymers for enhanced oil recovery (EOR) are polyacrylamide-based materials such as partially hydrolyzed polyacrylamide (HPAM). Such materials are relatively inexpensive, easily obtained, and perform fairly well in EOR applications. Specifically, HPAM is commonly used in polymer flooding because it provides good control over viscosity and effective permeability. However, it is widely recognized that HPAM is limited by poor thermal and mechanical stability. Thus, it would be extremely beneficial (in terms of application performance) to minimize shear degradation of the polymer backbone, especially at the high temperatures and high salinities characteristic of oil reservoirs.

Important characteristics of polymeric materials for EOR include good viscosity modification ability (achieved through water solubility, high molecular weight averages and the incorporation of carboxylate ions), reasonable chemical stability (achieved by incorporating high levels of amide groups into the polymer), and a good distribution of ions along the polymer backbone (that is, a targeted sequence length distribution). HPAM (which is essentially a copolymer of acrylamide (AAm) and acrylic acid (AAc)) meets these requirements, but the thermal and shear stability concerns described above must also be addressed. Therefore, a third comonomer, 2-acrylamido-2methylpropane sulfonic acid (AMPS) can be added to the polymer formulation, as the bulky sulfonic acid groups are expected to improve thermal stability and protect the main chain from 
shear degradation [1,2]. When a multi-component polymer like AMPS/AAm/AAc is being considered for any application, understanding and manipulating ternary reactivity ratios (which are related to both the cumulative terpolymer composition and the sequence length distribution) are essential [3].

Therefore, relationships between (experimental) synthesis conditions and AMPS/AAm/AAc terpolymer properties have been researched, verified and exploited. Recently, a comprehensive study was performed to establish the effect of synthesis conditions (like $\mathrm{pH}$, ionic strength, monomer concentration, and feed composition) on the terpolymerization kinetics and product terpolymer properties [4]. Careful design of experiments and subsequent analysis made it possible to establish that the key factors within the experimental range studied were ionic strength (which affects cumulative terpolymer composition and sequence length distribution), monomer concentration (which affects molecular weight averages) and feed composition (which, of course, impacts the cumulative composition of the terpolymer product).

Given the dependence of AMPS/AAm/AAc terpolymerization kinetics on the prepolymerization 'recipe', every effort was made so that the terpolymers described in this work be synthesized with consistent formulations. In an attempt to create materials that have desirable properties for the EOR application, the experimental conditions selected have been informed by the results of prior work [4]:

- The selected $\mathrm{pH}$ (at which synthesis occurs) for the optimally designed experiments is $\mathrm{pH}$ 7. In the previous study [4], no clear correlation between $\mathrm{pH}$ and reactivity ratio estimates was observed for the range of $\mathrm{pH} 5$ to $\mathrm{pH}$ 9. However, since acidic comonomers 
seem to be affected by changes in $\mathrm{pH}$, it is still important to select a $\mathrm{pH}$ for synthesis and adjust the pre-polymerization solution accordingly. A solution $\mathrm{pH}$ of 7 was selected herein because the condition is moderate (neutral) and because it allows for a direct extension of prior work [4].

- The optimal ionic strength (for the typical range of conditions used) was found to be 0.9 M. Lower ionic strength (IS) promotes the increased incorporation of acrylamide [4], and since high acrylamide content is desirable for the application, the low IS level (0.9 M) is more suitable for the synthesis of AMPS/AAm/AAc terpolymers for EOR.

- We have found that monomer concentration has a limited impact on the reactivity ratios for the AMPS/AAm/AAc terpolymerization conditions studied thus far [4]. By association, the terpolymer composition and microstructure were also minimally affected. However, as expected from polymerization kinetics, increasing the monomer concentration led to increased molecular weight averages. Since polymeric materials with high molecular weights (on the order of $10^{6} \mathrm{~g} / \mathrm{mol}$ ) are desirable for the EOR application, a total monomer concentration of $1.5 \mathrm{M}$ was used for each formulation.

- Under these conditions ( $\mathrm{pH} 7, \mathrm{IS}=0.9 \mathrm{M},[\mathrm{M}]=1.5 \mathrm{M}$ ), reactivity ratio estimates for the terpolymerization of AMPS/AAM/AAc are as shown in Table 1.

Table 1: Reactivity Ratio Estimates for the AMPS/AAm/AAc Terpolymer (from [3])

\begin{tabular}{|l|l|l|l|l|l|}
\hline rAMPS/AAm $_{\text {A }}$ & rAAm/AMPS & rAMPS/AAc $_{\text {A }}$ & rAA/AMPS & rAAm/AAc $_{\text {A }}$ & rAAC/AAm \\
\hline 0.66 & 0.82 & 0.82 & 0.61 & 1.61 & 0.25 \\
\hline
\end{tabular}


- Finally, two optimal feed compositions (both rich in acrylamide) were selected to allow for the most desirable terpolymer microstructure, as predicted by the AMPS/AAm/AAc reactivity ratios (as shown in Table 1 and determined in prior work [3,4]). Previously, we established that optimal terpolymers for EOR would have minimal 'blocky' sections and that the charge (from the acidic comonomers) would be well-distributed along the backbone. Optimizing model predictions (as described in [4]) indicates that feed compositions of $f_{\mathrm{AMPS}, 0} / f_{\mathrm{AAm}, 0} / f_{\mathrm{AAc}, 0}=0.21 / 0.69 / 0.10$ and $0.10 / 0.75 / 0.15$ will yield terpolymers with desirable properties.

An improved understanding of how reaction conditions affect polymerization kinetics and resulting polymer properties (including cumulative terpolymer composition, molecular weight averages, sequence length distribution, thermal stability, and rheological properties) provides the background information required for the design of optimal polymers for enhanced oil recovery. This is one step in a sequential, iterative design approach that will yield materials with targeted (and improved) application performance. In future work, the materials designed, synthesized and characterized herein will be further tested in terms of oil recovery performance and other application-specific requirements. However, developing and confirming these preliminary relationships is a necessary step within the framework of polymeric material design.

\section{Experimental Methodology}

\subsection{Materials}


Monomers 2-acrylamido-2-methylpropane sulfonic acid (AMPS; 99\%), acrylamide (AAm; electrophoresis grade, 99\%), and acrylic acid (AAc; 99\%) were purchased from Sigma-Aldrich (Oakville, ON, Canada). AAc was purified via vacuum distillation at $30^{\circ} \mathrm{C}$, while AAm and AMPS were used as received. Initiator (4,4'-azo-bis-(4-cyanovaleric acid), ACVA), inhibitor (hydroquinone) and sodium hydroxide were also purchased from Sigma-Aldrich. Sodium chloride from EMD Millipore (Etobicoke, ON, Canada) was used as received. In terms of solvents, water was Millipore quality (18 M $\Omega \cdot \mathrm{cm})$; acetone (99\%) and methanol (99.8\%) were used as received. Nitrogen gas (4.8 grade) used for degassing solutions was purchased from Praxair (Mississauga, ON, Canada).

\subsection{Polymer Synthesis}

Terpolymers of AMPS/AAm/AAc were synthesized under consistent solution properties at the levels determined from the definitive screening design results [4]. Sodium chloride was added to adjust ionic strength to $0.9 \mathrm{M}$, and all monomer solutions were titrated with sodium hydroxide to adjust the $\mathrm{pH}$ to approximately $7( \pm 0.5)$. Total monomer concentration was $1.5 \mathrm{M}$ for each synthesis, with $0.009 \mathrm{M}$ initiator (ACVA). Two optimal feed compositions (both rich in acrylamide) were selected to allow for the most desirable terpolymer microstructure (see Table 2). These feed compositions were based on predictions made using recently estimated reactivity ratios [4]. 
Table 2: Optimally Designed Experiments for Terpolymerization of AMPS/AAm/AAc

\begin{tabular}{|l|l|l|l|l|}
\hline Formulation & $\mathrm{pH}$ & $\mathrm{IS}$ & {$[\mathrm{M}]$} & Feed Composition $\left(\mathrm{f}_{\mathrm{AMPS}, 0} / \mathrm{f}_{\mathrm{AAm}, 0} / \mathrm{f}_{\mathrm{AAc}, 0}\right)$ \\
\hline$\# 1$ & 7 & $0.9 \mathrm{M}$ & $1.5 \mathrm{M}$ & $0.21 / 0.69 / 0.10$ \\
\hline$\# 2$ & 7 & $0.9 \mathrm{M}$ & $1.5 \mathrm{M}$ & $0.10 / 0.75 / 0.15$ \\
\hline
\end{tabular}

All solutions were degassed with $200 \mathrm{~mL} / \mathrm{min}$ nitrogen for $2 \mathrm{~h}$ prior to polymerization. After degassing, aliquots of $\sim 20 \mathrm{~mL}$ of solution were transferred to sealed vials using the cannula transfer method. Free-radical solution (aqueous phase) polymerizations were run in a temperaturecontrolled shaker-bath (OLS200; Grant Instruments, Cambridge, UK) at $40^{\circ} \mathrm{C}$ and $100 \mathrm{rpm}$. Vials were removed at selected time intervals, placed in ice and further injected with approximately 1 $\mathrm{mL}$ of $0.2 \mathrm{M}$ hydroquinone solution to stop the polymerization. Polymer samples were isolated by precipitating the products in acetone, filtered (paper filter grade number 41, Whatman; SigmaAldrich, Oakville, ON, Canada) and vacuum dried for 1 week at $50^{\circ} \mathrm{C}$. Both polymerizations were independently replicated.

\subsection{Characterization of Polymer Properties}

\subsubsection{Conversion and Composition}

Conversion of all polymer samples was determined using gravimetry. Due to the high ionic strength (and necessarily high salt content), we observed that some sodium chloride remained present in the polymer samples. This was initially deduced from elemental analysis results and uncharacteristically high conversion calculations, and then independently confirmed for select samples via inductively coupled plasma mass spectrometry (ICP). As per the recommendation of Riahinezhad et al. [5], the mass of the sodium ions (attracted to the dissociated acids along the polymer chain) was considered in conversion calculations. 
Polymer composition was measured using Elemental Analysis (CHNS, Vario Micro Cube, Elementar). The machine was calibrated daily (and after every 60 samples) using a sulfanilamide standard and samples were combusted at $1150^{\circ} \mathrm{C}$. The content of elemental $\mathrm{C}, \mathrm{H}, \mathrm{N}$ and $\mathrm{S}$ in the samples was determined, which allowed for the subsequent calculation of cumulative terpolymer composition. Composition calculations did not include $\mathrm{H}$ measurements, as residual water has been known to affect the determined $\mathrm{H}$ content.

\subsubsection{Molecular Weight}

Molecular weight averages were determined using gel permeation chromatography (PL-GPC 50, Agilent, with two columns, type PL aquagel-OH MIXED-H $8 \mu \mathrm{m}$, Agilent). To analyze the AMPS/AAm/AAc terpolymers, four detectors were employed: refractive index, low-angle and right-angle light scattering (LALLS/RALLS), and differential pressure. To minimize the charge interactions between the column and the polymer samples, a buffer solution of $\mathrm{pH} 7$ was used as the mobile phase (flowing at a rate of $1.0 \mathrm{~mL} / \mathrm{min}$ ). The buffer was prepared using sodium nitrate (0.2 M) and sodium phosphate (monobasic and dibasic, $0.1 \mathrm{M}$ ) in Millipore quality water. The synthesized polymers were dissolved in the mobile phase (pH 7 buffer) to obtain concentrations of $\sim 1 \mathrm{mg} / \mathrm{mL}$. The solution preparation step required fine grinding prior to dissolving polymers in buffer, and allowing the solutions to sit under ambient conditions (with occasional manual mixing) until the polymer was dissolved. Prior to injection, polymer solutions were filtered through a 0.2 $\mu \mathrm{m}$ filter. Polyacrylic acid - sodium salt (PAAc-Na) calibration standards obtained from Agilent Technologies were used to calibrate detector constants. For all unknown samples, a dn/dc of 0.175 was used (based on the dn/dc of the PAAc-Na standards, verified by laser refractometry). 
Molecular weight measurements rely on the hydrodynamic volume of polymer chains, which in turn are affected by additional factors. This is especially true for polyelectrolytes, as variations in cumulative composition, sequence length distribution, and ionic strength (charge effects) affect the coil conformation (and, by extension, the hydrodynamic volume). Additionally, for polymers with high hydrodynamic volumes, 'retardation' can occur alongside typical size exclusion fractionation [6]. It is well-established that this phenomenon can result in the underestimation of molecular weight averages (and, especially, underestimated polydispersity) [7]. Therefore, in the current work, peak average molecular weight $\bar{M}_{p}$ is the primary measure of molecular weight averages.

\subsubsection{Sequence Length Distribution}

${ }^{13} \mathrm{C}-\mathrm{NMR}$ (nuclear magnetic resonance) was conducted on a Bruker AVANCE 500 Ultrashield NMR spectrometer (Nuclear Magnetic Resonance Facility, Department of Chemistry, University of Waterloo). The NMR was run for 12 hours per sample at $68{ }^{\circ} \mathrm{C}$ (around 6,000 scans) and employed inverse gated proton decoupling (30 degree pulse) with a pulse delay of $6 \mathrm{~s}$ (D1 = $6 \mathrm{~s}$ ).

To prepare the samples for analysis, each terpolymer was dissolved in a $\mathrm{pH} 7 \mathrm{D}_{2} \mathrm{O} / \mathrm{buffer}$ solution (prepared using $0.2 \mathrm{M}$ sodium nitrate and $0.1 \mathrm{M}$ sodium phosphate (monobasic and dibasic) as for GPC, but with $\mathrm{D}_{2} \mathrm{O}$ as the mobile phase). Polymer samples were finely ground and slowly added to the buffer to achieve a concentration of $6 \mathrm{wt} \%$. Solutions were injected into NMR tubes using a long-tipped needle and a syringe, and tubes were heated in a $60^{\circ} \mathrm{C}$ water bath to ensure all bubbles had escaped prior to analysis. 


\subsubsection{Thermal Stability}

Select samples were analyzed using thermal gravimetric analysis (TGA) on a Q500 TGA from TA Instruments (Analytical Instrumentation Lab, Department of Chemical Engineering, University of Waterloo). Small sample quantities $(<5 \mathrm{mg})$ were placed in platinum pans for analysis, and samples were run from $30^{\circ} \mathrm{C}$ to $600^{\circ} \mathrm{C}$ with a heating rate of $10^{\circ} \mathrm{C} / \mathrm{min}$ under nitrogen atmosphere. It was especially important to use small amounts of sample ( $<5 \mathrm{mg})$, since entrapped water could result in sample expansion during analysis.

\subsubsection{Rheology}

Solutions for rheological analysis were prepared by dissolving finely ground terpolymer samples in Millipore quality water or pH 7 buffer (prepared as described for GPC; Section 2.3.2); solutions were made to have a concentration of $0.01 \mathrm{~g} / \mathrm{mL}$. A stress-controlled cone and plate rheometer (AR2000, TA Instruments) was used to measure the viscoelastic properties of the polymer solutions. An environmental test chamber (ETC) steel cone with $40 \mathrm{~mm}$ diameter and $1^{\circ}$ angle was used for all tests, and all measurements were taken at $25^{\circ} \mathrm{C}$.

\section{Results and Discussion}

Consistency of polymer properties (that is, good experimental reproducibility) is extremely important, since several samples will eventually be combined for the litmus test of polymer flooding/EOR testing [8]. For both optimal formulations described in Table 2, genuine replication was carefully incorporated into the synthesis and subsequent characterization. At the synthesis 
stage, terpolymers \#1 and \#1R (both of the same 'recipe', formulation \#1) were synthesized from two unique (independently prepared) monomer stock solutions. Similarly, terpolymers \#2 and \#2R were from independently prepared monomer stock solutions, and terpolymer \#2RB was synthesized from the same stock solution as \#2R. Thus, reproducibility between stock solutions (with the same target formulation) and repeatability within a given stock solution (using the same concentrated solution for two separate synthesis procedures) have been considered. In addition, characterization replicates were performed for the evaluation of terpolymer properties described in what follows.

\subsection{Cumulative Terpolymer Composition}

The cumulative terpolymer compositions for both optimal formulations were predicted from previously estimated ternary reactivity ratios $[3,4]$. We expected that the terpolymers would be rich in acrylamide and exhibit minimal composition drift throughout conversion. To confirm the cumulative terpolymer composition (and to evaluate the prediction performance of the reactivity ratio estimates), samples throughout the conversion trajectory were analyzed. The results (and comparisons to model predictions) are shown in Figure 1 and Figure 2. 


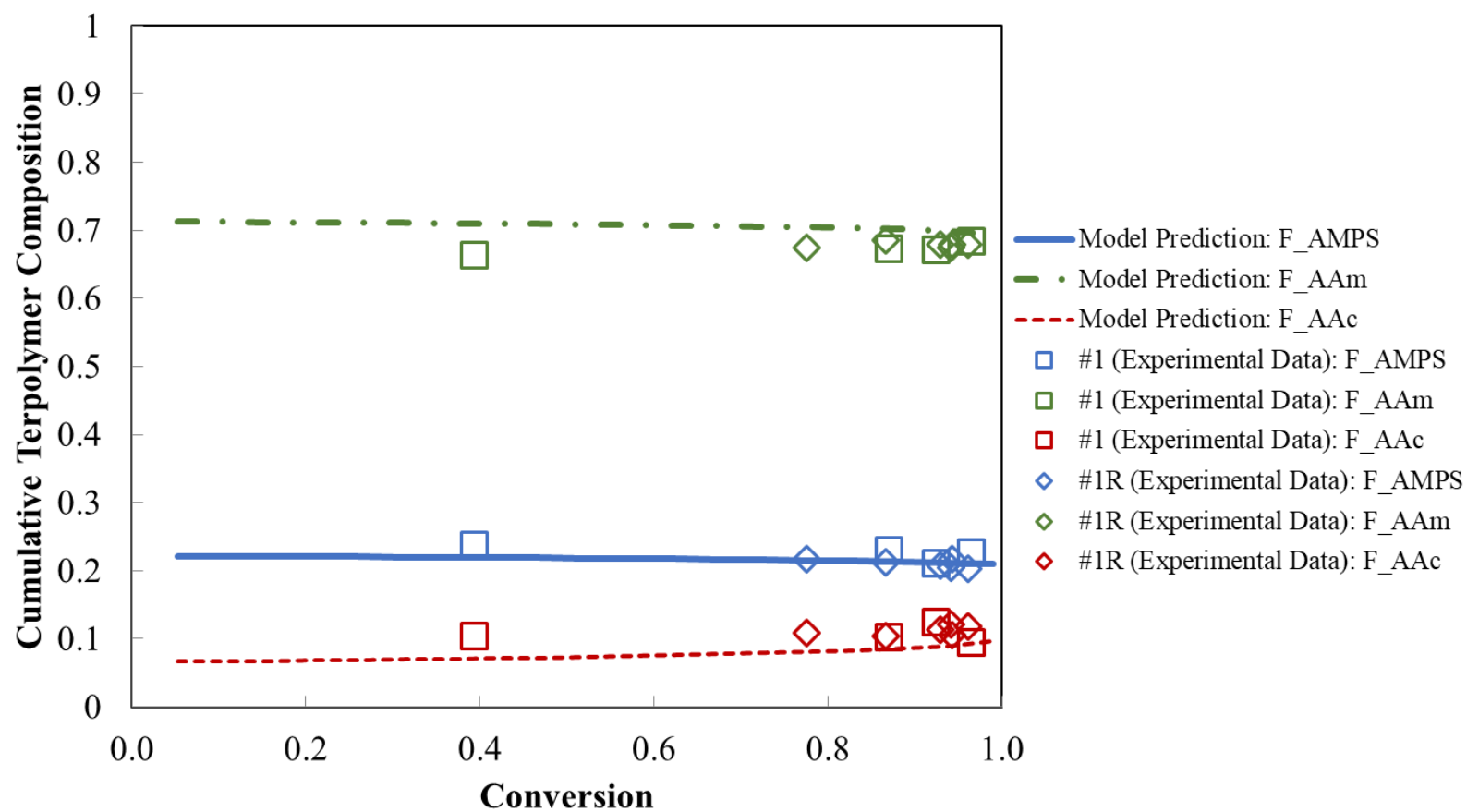

Figure 1: Cumulative Terpolymer Composition for Formulation \#1 $\left(f_{\mathrm{AMPS}, 0} / f_{\mathrm{AAm}, 0} / f_{\mathrm{AAc}, 0}=\right.$ $0.21 / 0.69 / 0.10)$

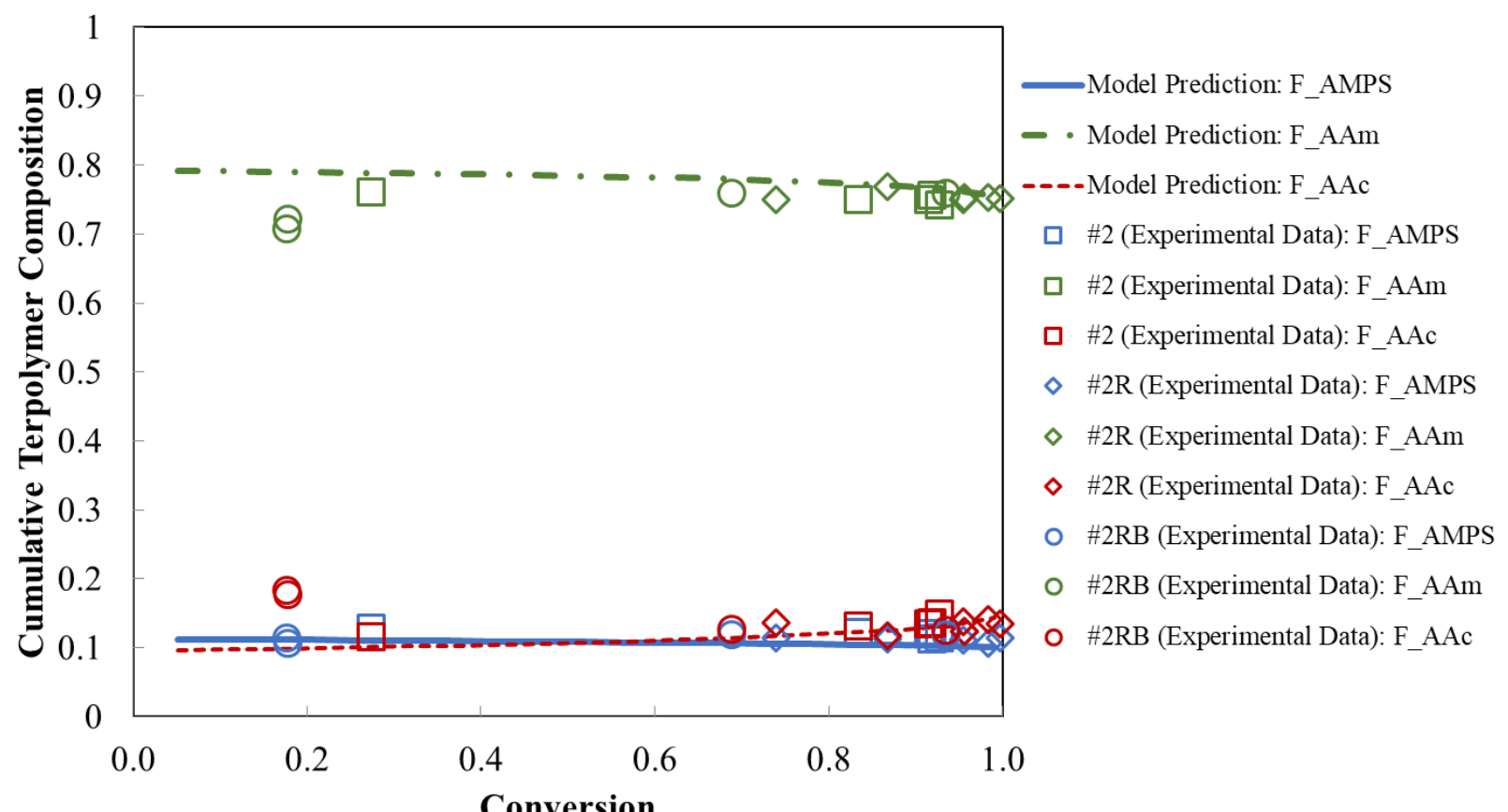

Figure 2: Cumulative Terpolymer Composition for Formulation \#2 $\left(f_{\mathrm{AMPS}, 0} / f_{\mathrm{AAm}, 0} / f_{\mathrm{AAc}, 0}=\right.$ $0.10 / 0.75 / 0.15)$ 
Experimental data collected from both formulations are in good agreement with the model predictions. Overall, the composition values are close to the predicted values, and composition drift is minimal. Some specific points at low conversion (at 18\% conversion, for example) are worth examining further. Interestingly, the (repeatable) elemental analysis measurement indicates that the AAc fraction exceeds the AMPS fraction, which conflicts with the model prediction. This could be due to small amounts of experimental error (likely propagating from the synthesis step, since the elemental analysis measurements were replicated). It is also possible that the behaviour of the terpolymerization varies at low conversion levels, and that some composition drift exists early in the polymerization process (below 20\% conversion). In this case, though, the discrepancy at $18 \%$ conversion is not a major concern. For the application, large quantities of material are desirable, so the polymerization process will typically be taken to higher conversion levels. The main conclusion here is that overall, our model predictions (from ternary reactivity ratios estimated earlier $[3,4])$ accurately predict the cumulative terpolymer composition.

\subsection{Molecular Weight Averages}

Given the results of the preliminary study [4], the expected range for the peak average molecular weights $\left(\bar{M}_{p}\right)$ of these samples was between $1.0 \times 10^{6} \mathrm{~g} / \mathrm{mol}$ and $5.0 \times 10^{6} \mathrm{~g} / \mathrm{mol}$. For both formulations, excellent repeatability was observed. This is true for a variety of comparisons: between two independently synthesized polymers with the same formulation (for example, terpolymer \#1 samples vs. terpolymer \#1R samples), between samples independently isolated during a common synthesis (for example, \#1-4 vs. \#1-7; both taken after 90 minutes of polymerization), and between characterization tests (two GPC replicates of \#1-4, completed over several months). Relevant data are shown in the Supporting Information (Table S1 and Table S2). 
No statistically significant differences were observed for any of the comparisons. Therefore, the synthesis replicates and the sampling replicates do not significantly contribute to overall variability.

Aside from examining reproducibility/repeatability, we can also evaluate the average molecular weights of each sample. For formulation $\# 1\left(f_{\mathrm{AMPS}, 0} / f_{\mathrm{AAm}, 0} / f_{\mathrm{AAc}, 0}=0.21 / 0.69 / 0.10\right)$, the (mean) peak average molecular weight is $1.57 \times 10^{6} \mathrm{~g} / \mathrm{mol}$. Formulation $\# 2\left(f_{\mathrm{AMPS}, 0} / f_{\mathrm{AAm}, 0} / f_{\mathrm{AAc}, 0}=\right.$ $0.10 / 0.75 / 0.15)$ is about the same, with a mean $\bar{M}_{p}$ of $1.51 \times 10^{6} \mathrm{~g} / \mathrm{mol}$. The similarity allows for straightforward comparison of other (application-specific) properties; any differences in performance will be due to other factors (such as cumulative terpolymer composition or terpolymer microstructure).

The molecular weight averages reported here are within the anticipated desired range. Also, these results are well-aligned with a reference polymer that is currently used as a commercially available product in EOR applications. The reference polymer is an acrylamide/acrylic acid copolymer (with cumulative mole fractions of $\bar{F}_{A A m}=0.91$ and $\bar{F}_{A A C}=0.09$ ), and the peak average molecular weight is $1.42 \times 10^{6} \mathrm{~g} / \mathrm{mol}$. Therefore, we anticipate that the application performance will be similar.

For interest, we also examined how molecular weight data vary with conversion. Although most data collected were for high conversion samples, the samples taken at low conversion levels indicate that the peak average molecular weight (Figure 3a) and the bulk intrinsic viscosity (IV; 
Figure 3b) are relatively constant over the conversion range analyzed (approximately 15\% to $100 \%)$

Both plots related to the molecular weight analysis (within Figure 3) also include the properties of the reference polymer. Since the conversion of the reference sample is unknown (but likely very high), the measured value for each property is presented as a horizontal (dashed) line. Both of the newly synthesized (optimal) terpolymers have similar molecular weight characteristics to the reference material, which suggests that our customized (designed) approach led to the development of materials with appropriate and desirable molecular weight characteristics. Also, the peak average molecular weights of the new materials are slightly higher than that of the reference material, which is advantageous for EOR performance. 
(a)

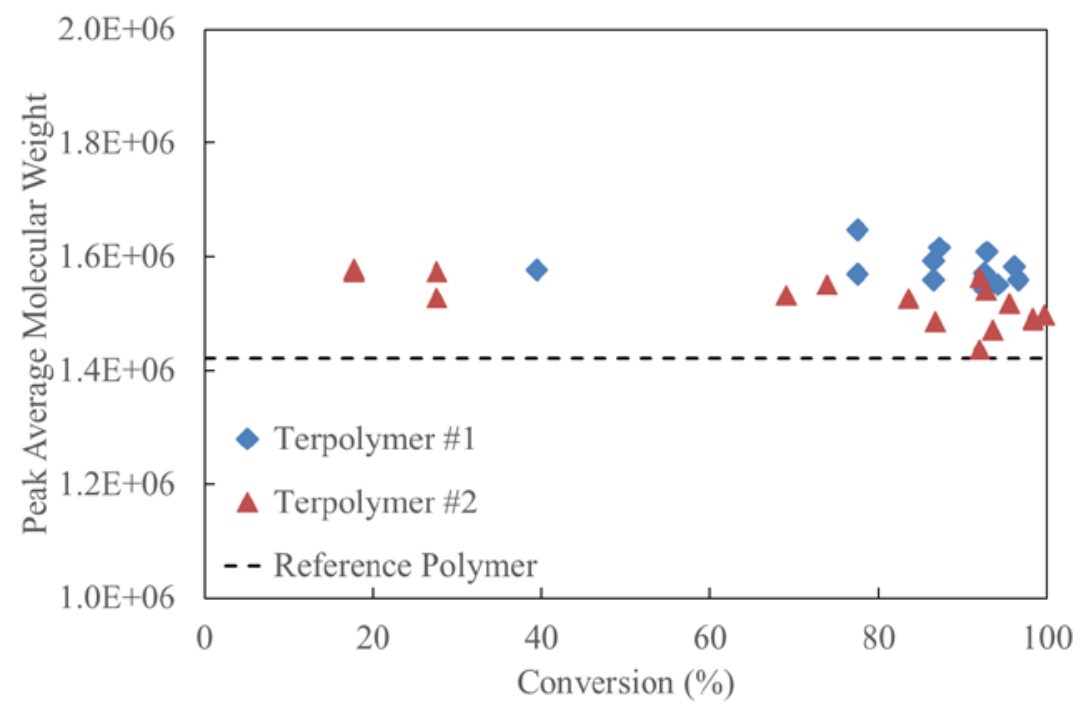

(b)

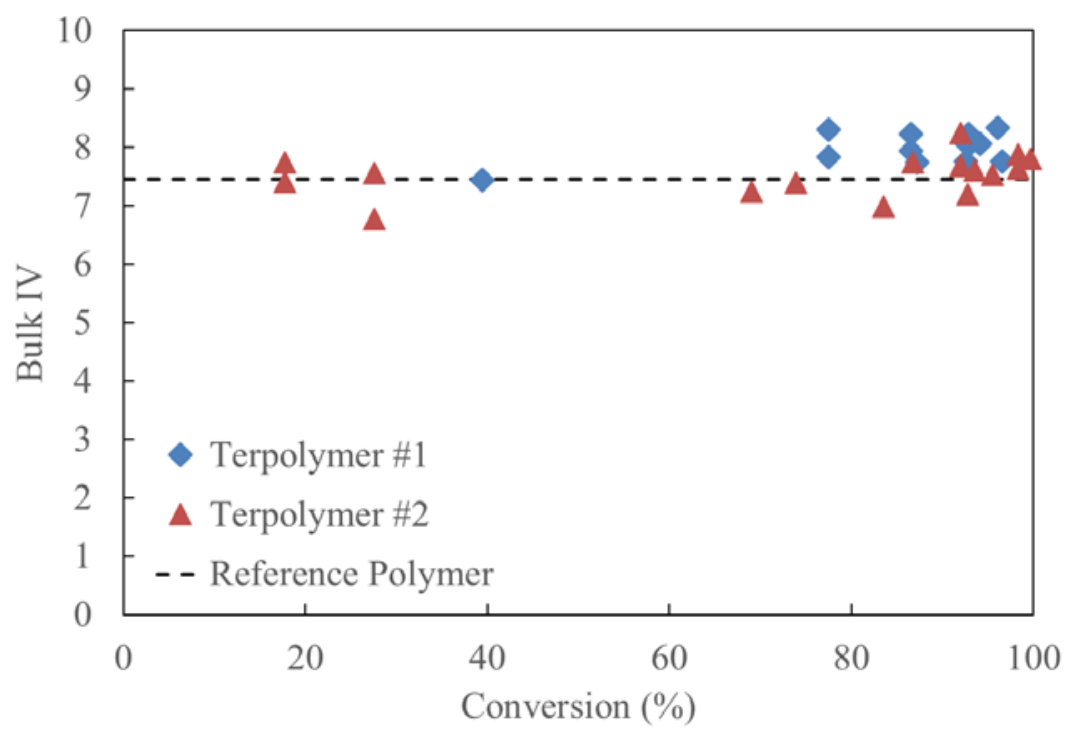

Figure 3: Effect of Conversion on (a) Peak Average Molecular Weight and (b) Bulk Intrinsic Viscosity (and Comparison to Reference Polymer) for Optimal Terpolymers

\subsection{Sequence Length Distribution}

${ }^{13} \mathrm{C}-\mathrm{NMR}$ spectra were measured and analyzed as described in Section 2.3.3. The purpose of these tests was to compare the measured sequence length distributions to those predicted by ternary reactivity ratios (using analysis techniques similar to Randall [9] and Brar and Sunita [10]). 
However, given the 18 unique monomer triads, the chemical similarity of the comonomers, and the noisy spectra (due to the high viscosity of the samples), sequence length distribution could not be directly determined. For reference, ${ }^{13} \mathrm{C}-\mathrm{NMR}$ spectra of terpolymer \#1 and terpolymer \#2 are presented in Figure 4 and Figure 5, respectively.

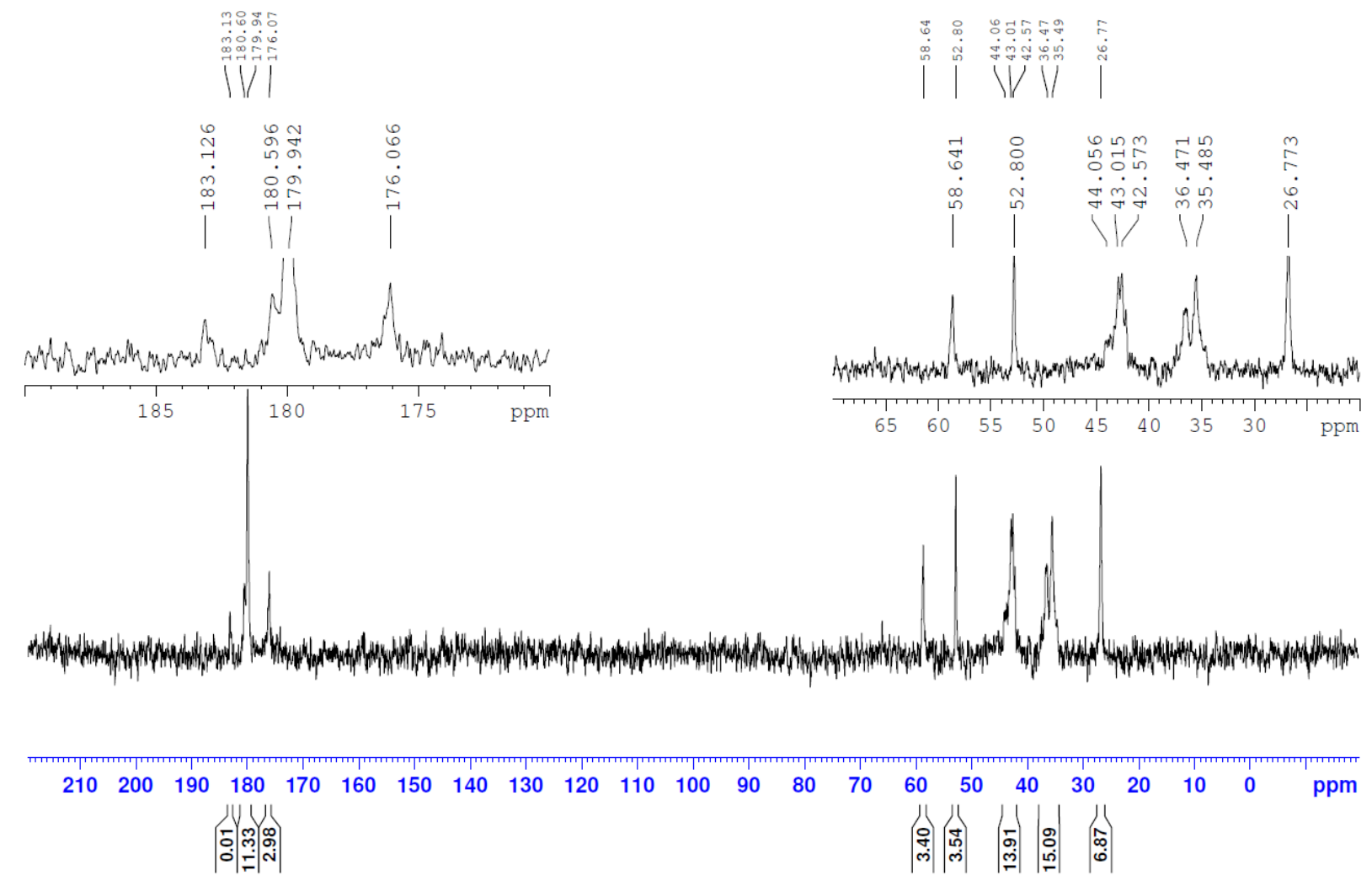

Figure 4: ${ }^{13} \mathrm{C}$-NMR Spectra of Terpolymer $\# 1$ in Buffer/ $\mathrm{D}_{2} \mathrm{O}$ at $68^{\circ} \mathrm{C}$ 


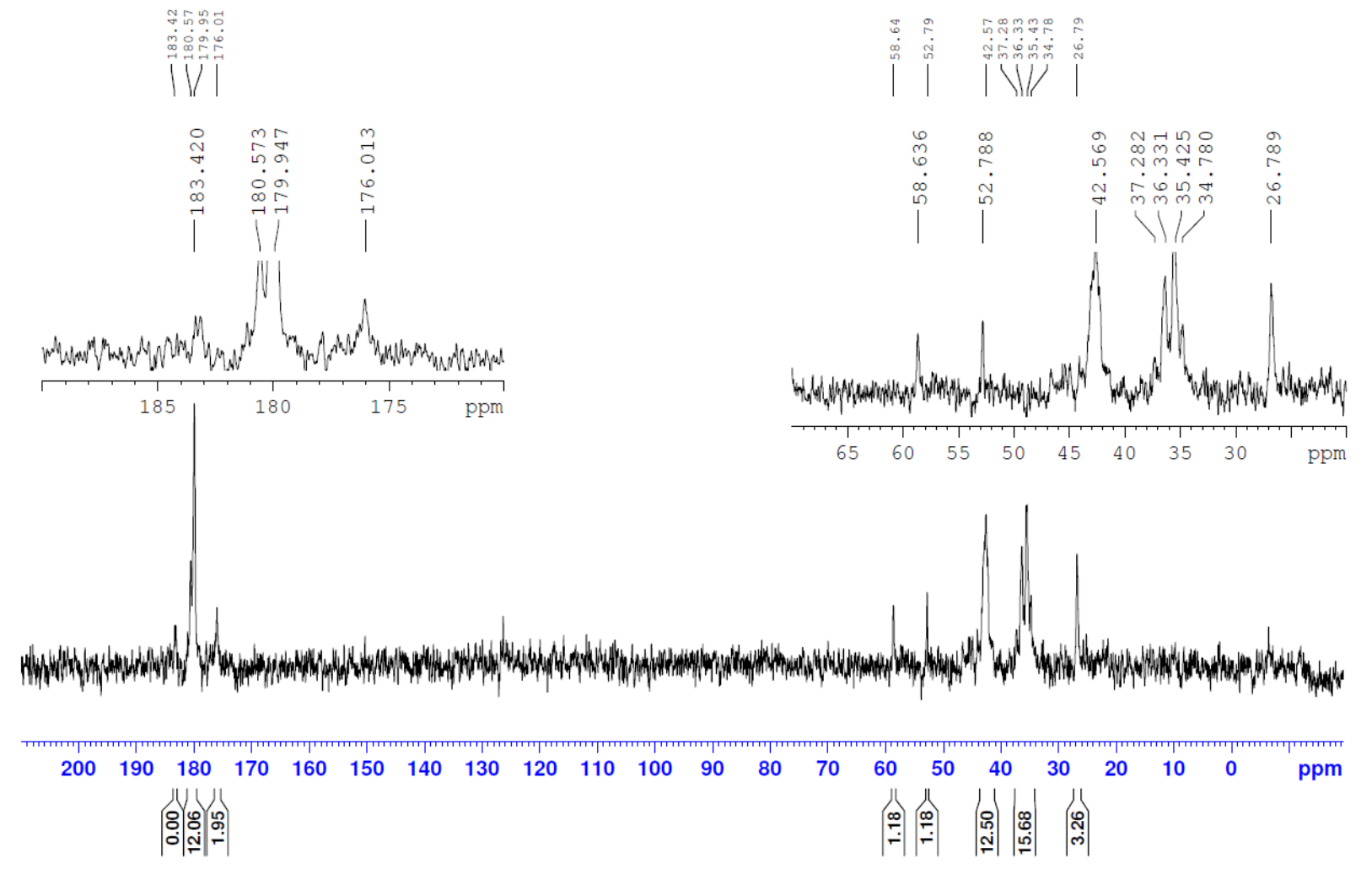

Figure 5: ${ }^{13} \mathrm{C}-\mathrm{NMR}$ Spectra of Terpolymer $\# 2$ in Buffer/ $\mathrm{D}_{2} \mathrm{O}$ at $68^{\circ} \mathrm{C}$

The spectra can be used to calculate the cumulative terpolymer composition. Using the carbonyl carbon responses associated with AMPS $(\delta \approx 176 \mathrm{ppm})$, AAm $(\delta \approx 180 \mathrm{ppm})$ and AAc $(\delta \approx 183$ ppm), we can calculate the mole fraction of each comonomer in the terpolymer sample. These are compared to the (averaged) elemental analysis results in Table 3. 
Table 3: Comparison of Cumulative Terpolymer Composition from ${ }^{13} \mathrm{C}-\mathrm{NMR}$ and Elemental Analysis

\begin{tabular}{|l|c|c|c|c|c|c|}
\hline & \multicolumn{3}{|c|}{$\begin{array}{c}\text { Cumulative Composition from }{ }^{13} \text { C- } \\
\text { NMR }\end{array}$} & \multicolumn{3}{c|}{$\begin{array}{c}\text { Cumulative Composition from } \\
\text { Elemental Analysis }\end{array}$} \\
\hline & $\bar{F}_{A M P S}$ & $\bar{F}_{A A m}$ & $\bar{F}_{A A C}$ & $\bar{F}_{A M P S}$ & $\bar{F}_{A A m}$ & $\bar{F}_{A A C}$ \\
\hline Terpolymer \#1 & 0.19 & 0.74 & 0.07 & 0.21 & 0.68 & 0.11 \\
\hline Terpolymer \#2 & 0.13 & 0.80 & 0.07 & 0.11 & 0.75 & 0.13 \\
\hline
\end{tabular}

Composition measurements are in relatively good agreement between ${ }^{13} \mathrm{C}$-NMR and elemental analysis, especially considering the challenges associated with analyzing viscous polymer samples using nuclear magnetic resonance. ${ }^{13} \mathrm{C}-\mathrm{NMR}$ results provide adequate confirmation of the elemental analysis results, but the elemental analysis results are more trustworthy.

\subsection{Thermal Stability}

Thermal gravimetric analysis (TGA) was performed (as described in Section 2.3.4) for both terpolymer formulations, and included synthesis replicate analysis and characterization replicate analysis. The reference material and an additional AAm/AAc copolymer (from Riahinezhad et al. [11]) were also evaluated for comparison. The motivation here was two-fold. First, it was important to ensure that the thermal properties were consistent across synthesis replicates (and to ensure that TGA measurements were consistent for sample replicates). Second, thermal analysis provides an opportunity to confirm that thermal stability is improved (compared to the typically used AAm/AAc copolymer) when AMPS is incorporated into the polymer backbone [12-15]. The majority of oil reservoirs are below $200^{\circ} \mathrm{C}$, but behaviour at higher temperatures was evaluated 
herein as a 'worst case' scenario. TGA up to higher temperatures (at least $400^{\circ} \mathrm{C}$ ) made it possible to compare the point at which significant mass loss occurred for each sample; materials that showed degradation at higher temperatures are more thermally stable (and may, therefore, be promising candidates for EOR). Results from synthesis replicates (\#1 vs. \#1R and \#2 vs. \#2R), the reference material and the (previously synthesized) AAm/AAc copolymer are shown in Figure 6.

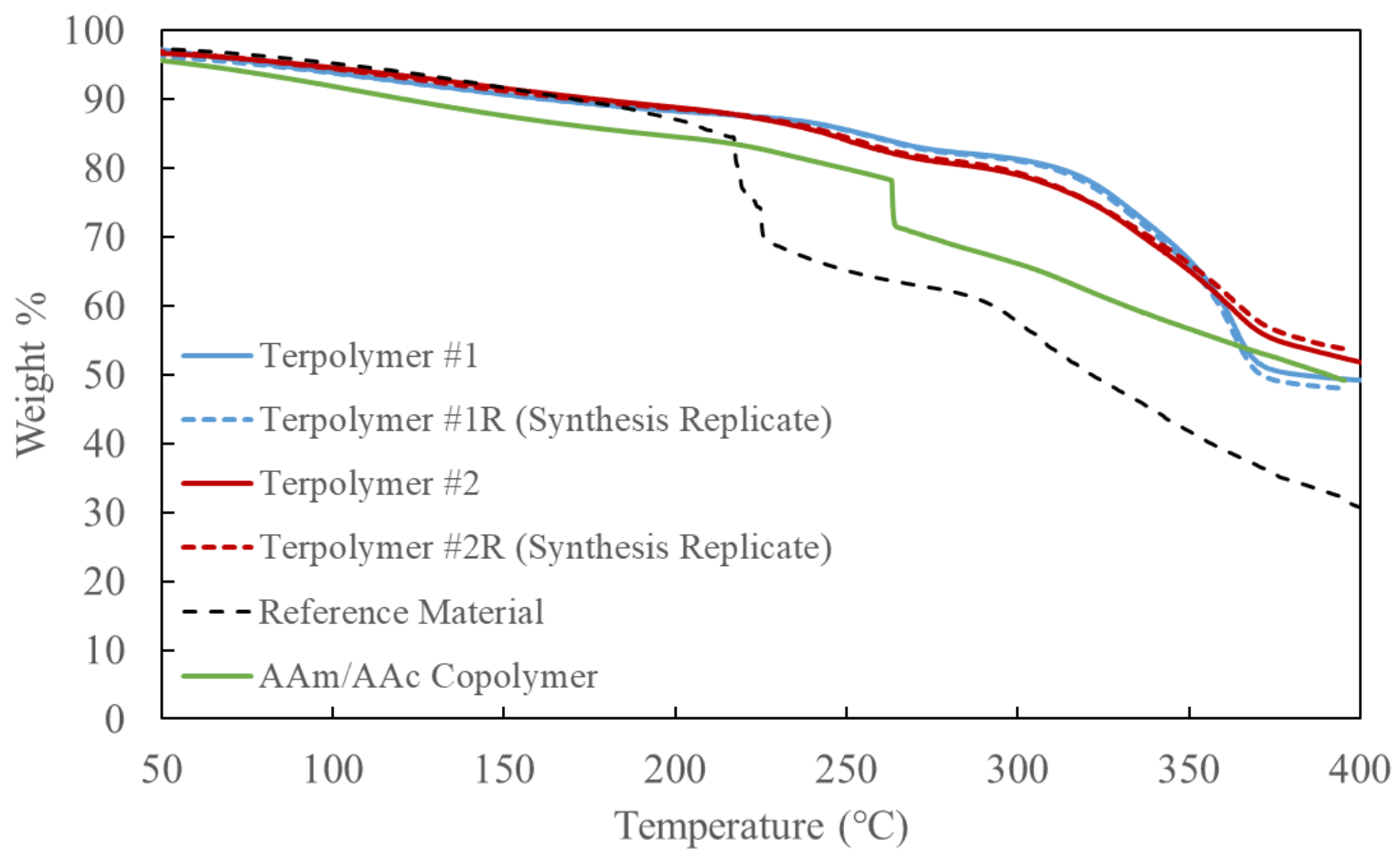

Figure 6: Thermal Behaviour (from TGA) for Optimally Designed Terpolymers, Reference Material, and an AAm/AAc Copolymer

Examining all six curves on a single plot allows for the direct comparison of all materials. Immediately, we see excellent agreement between the original runs and the synthesis replicates. Both blue curves (for terpolymers \#1 and \#1R) are directly on top of each other, and exhibit two main points of interest. Aside from the gradual decrease in weight initially (which is likely water loss), there are two more obvious transition points. The first transition occurs at $225^{\circ} \mathrm{C}$ and the 
second (more significant) transition occurs at $284^{\circ} \mathrm{C}$. Thus, any substantial mass loss (degradation) is beyond $200^{\circ} \mathrm{C}$. Similarly, excellent repeatability was observed from the independent replication of terpolymer \#2. Both red curves exhibit similar behaviour, and the transition points are identical.

Another important conclusion we can draw from Figure 6 is the improved thermal behaviour of the terpolymers compared to the reference material and to the AAm/AAc copolymer. Both of these materials show a sudden decrease in sample mass: the reference material at $217^{\circ} \mathrm{C}$ and the AAm/AAc copolymer at $257^{\circ} \mathrm{C}$. The mass reduction for these materials is much more sudden than for the terpolymers; this could have adverse effects in the EOR application.

Table 4 provides some key findings from the TGA experimental work. A minor transition (with small mass loss) was only observed for the terpolymer samples, and may be related to water entrapment in the polymeric materials. The major transition is the point at which significant mass loss occurs, and is likely related to sample degradation. The major transition occurs at the lowest temperature for the reference material; the previously synthesized AAm/AAc copolymer can tolerate an additional $40^{\circ} \mathrm{C}$ before the major transition occurs. Even more improvement is observed when AMPS is added to the material formulation, as both terpolymer formulations (\#1 and \#2) exhibit (relatively low) weight loss at $284^{\circ} \mathrm{C}$ and $281^{\circ} \mathrm{C}$, respectively.

It is also enlightening to examine the remaining weight proportion at several (meaningful) temperatures. In Table 4 , the remaining weight $\%$ of each material is listed at $80^{\circ} \mathrm{C}$ (median reservoir temperature as per [16]), $120^{\circ} \mathrm{C}$ (maximum 'encountered' reservoir temperature for $\sim 90 \%$ of reservoirs worldwide, again as per [16]), $200^{\circ} \mathrm{C}$ (maximum reservoir temperature) and 
$300^{\circ} \mathrm{C}$ (for interest and to represent a 'worst case scenario'). For replicated samples, average values are shown.

Table 4: Points of Interest from TGA Results

\begin{tabular}{|l|c|c|c|c|c|c|}
\hline Material & $\begin{array}{c}\text { Minor } \\
\text { Transition }\end{array}$ & $\begin{array}{c}\text { Major } \\
\text { Transition }\end{array}$ & $\begin{array}{c}\text { Weight \% } \\
\text { at } 80^{\circ} \mathrm{C}\end{array}$ & $\begin{array}{c}\text { Weight \% } \\
\text { at } 120^{\circ} \mathrm{C}\end{array}$ & $\begin{array}{c}\text { Weight \% } \\
\text { at } 200^{\circ} \mathrm{C}\end{array}$ & $\begin{array}{c}\text { Weight \% } \\
\text { at } 300^{\circ} \mathrm{C}\end{array}$ \\
\hline Terpolymer \#1 & $225^{\circ} \mathrm{C}$ & $284^{\circ} \mathrm{C}$ & $95.4 \%$ & $92.8 \%$ & $88.2 \%$ & $80.8 \%$ \\
\hline Terpolymer \#2 & $199^{\circ} \mathrm{C}$ & $281^{\circ} \mathrm{C}$ & $95.6 \%$ & $93.3 \%$ & $88.7 \%$ & $79.1 \%$ \\
\hline $\begin{array}{l}\text { Reference } \\
\text { Copolymer }\end{array}$ & N/A & $217^{\circ} \mathrm{C}$ & $96.2 \%$ & $94.0 \%$ & $87.1 \%$ & $57.7 \%$ \\
\hline $\begin{array}{l}\text { AAm/AAc } \\
\text { Copolymer }\end{array}$ & N/A & $257^{\circ} \mathrm{C}$ & $93.5 \%$ & $90.0 \%$ & $84.5 \%$ & $66.1 \%$ \\
\hline
\end{tabular}

All materials exhibit similar thermal behaviour up to $200^{\circ} \mathrm{C}$, but the difference in material properties becomes evident when we examine measurements at $300^{\circ} \mathrm{C}$. In reality, thermal stability up to $300^{\circ} \mathrm{C}$ is much higher than what the EOR application currently demands, but the contrast in materials shows the improved thermal stability when a terpolymer of AMPS/AAm/AAc is used. Thus, we have confirmed through this study that the addition of AMPS does, in fact, improve the thermal stability of the polymeric material.

\subsection{Rheological Properties}

Rheological properties are important for EOR performance. If the polymer solutions are not viscous enough, they will not provide the sweep efficiency required (that is, much of the residual oil will remain in the reservoir, even after polymer flooding). In contrast, if they are too viscous, they may cause pressure build-up and plugging in the reservoir. There is potential to adjust the 
viscosity of a solution by changing the concentration of the polymeric material within the solution, but using smaller quantities of the polymer is preferred (for environmental and economic reasons). Therefore, the goal is to create materials with properties that are similar to (or better than) the reference material. Rheological properties of each optimal terpolymer and the reference polymer (in water and in a pH 7 buffer solution) were measured as described in Section 2.3.5, and key findings are presented herein.

Frequency sweep tests, performed using a cone and plate rheometer, give information about the viscoelastic properties of the polymer solution. However, prior to completing each frequency sweep test, the linear viscoelastic region (LVR) must be established via a strain sweep test. Strain sweep tests were conducted at a fixed frequency of $10 \mathrm{~Hz}$, ranging from $0.1 \%$ to $20 \%$; a representative result for a (formulation \#1) terpolymer sample in water is shown in Figure 7. This is representative of the solutions in the present study, and $1 \%$ strain was chosen for subsequent frequency sweep testing.

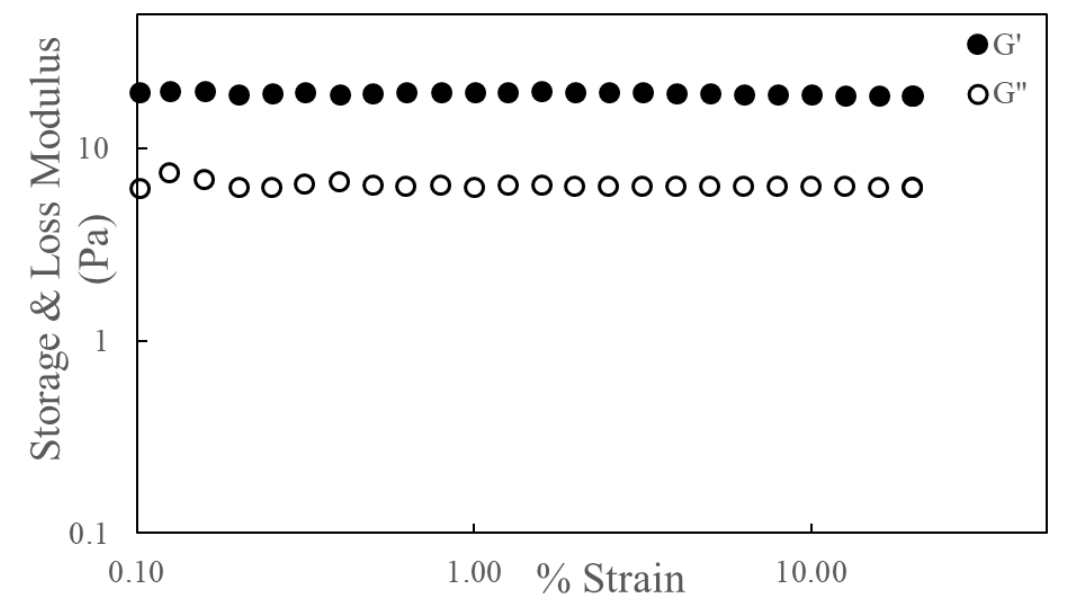

Figure 7: Sample Results (from Terpolymer \#1) for Strain Sweep Test at $10 \mathrm{~Hz}$ (solution concentration of $0.01 \mathrm{~g} / \mathrm{mL}$ in water) 
Representative plots (including replicates) from frequency sweep tests are shown in Figure 8. The shear thinning behaviour of the AMPS/AAm/AAc terpolymer solutions is immediately obvious: samples show a decrease in viscosity as angular frequency increases. This makes physicochemical sense, since at higher frequencies (or by analogy, at higher shear rates), the polymers transition from flowing in a coil conformation to flowing in a linear (aligned) arrangement. This decreases the viscosity of the solution, which in turn would decrease the efficiency of the polymer flooding process.

We can also examine the repeatability of the experimental results (both in terms of synthesis and characterization). For terpolymer \#1, the synthesis replicates seem to exhibit more inconsistencies than the characterization replicates (especially for terpolymer \#1 in water; Figure 8a), but this is often expected. For terpolymer \#2, very good agreement is observed between synthesis replicates.

For the rheological tests done in water, the complex viscosities of both optimal terpolymers were higher than the reference sample at low angular frequencies, and over most of the angular frequency range studied. However, this distinction became less pronounced at higher angular frequencies (especially for terpolymer \#1R (Figure 8a) and for terpolymer \#2 (Figure 8b)).

We also examined the change in behaviour between aqueous polymer solutions and polymers in buffer solutions. For the reference polymer and for our newly synthesized (optimal) terpolymers, the complex viscosity is lower in buffer solutions than in water. Physicochemically, these results are as expected. The terpolymer of AMPS/AAm/AAc is a polyelectrolyte and is therefore very sensitive to ions in solution. When charged molecules (salts, in this case) are added to the 
environment, charge screening occurs. As a result, previously expanded polymer chains (resulting from charge repulsion along the macromolecule) reposition themselves into a tighter coil conformation. Of course, this change in polymer conformation impacts the solution viscosity; a smaller radius of gyration will lower the shear viscosity of a given polymer solution.

Interestingly, our designed terpolymers exhibited a larger reduction in complex viscosity (compared to the reference material) when the buffer solution was used rather than water. This is likely due to the addition of AMPS; incorporating a second acidic comonomer into the polymeric material amplifies the charge effects. However, it is anticipated that the presence of AMPS will have additional benefits, including mechanical and chemical stability (as per [1,2] and the earlier discussion herein). Also, the rheological behaviour of both terpolymer formulations is comparable to the reference material, which makes them good candidates for the EOR application. 
(a)

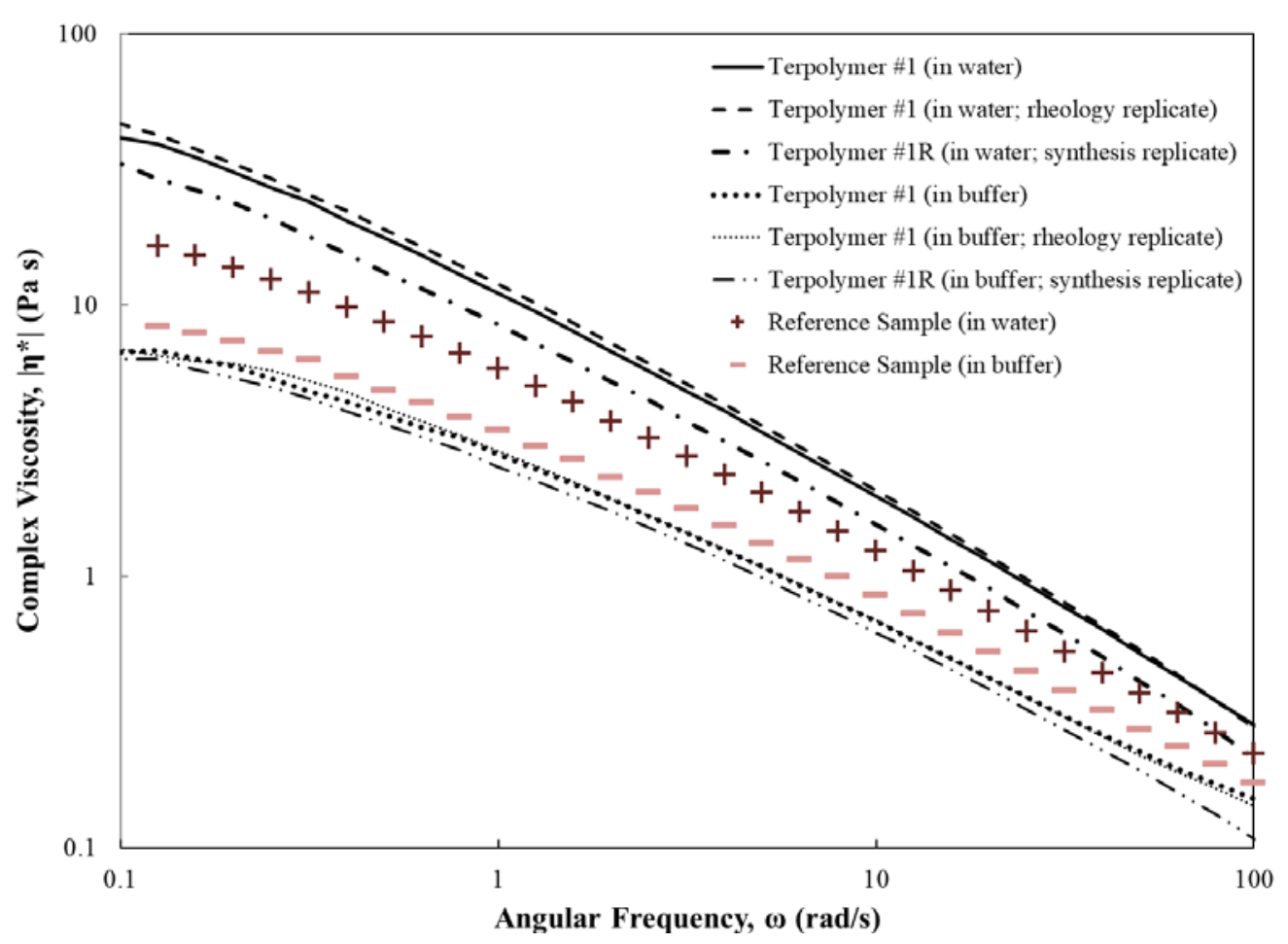

(b)

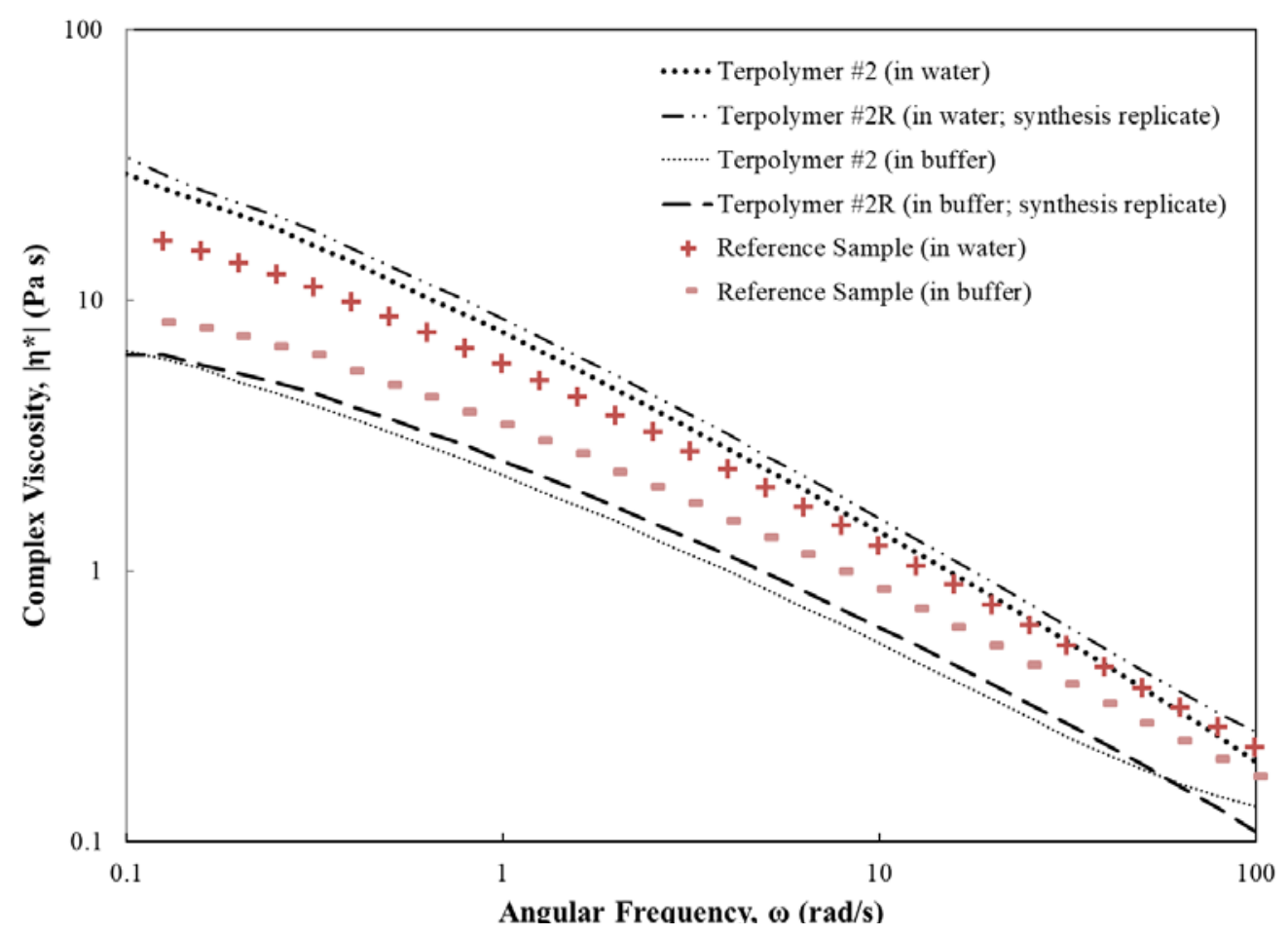

Figure 8: Complex Viscosity Profiles for AMPS/AAm/AAc Terpolymers in Water and Buffer

for (a) Terpolymer \#1 and (b) Terpolymer \#2 
It has been reported that the polymer flood water solutions used in EOR are exposed to a range of shear rates from about $1 \mathrm{~s}^{-1}$ to $7 \mathrm{~s}^{-1}$ [11]. The Cox-Merz rule allows us to assume that the relationship between $\left|\eta^{*}\right|$ and $\omega$ is analogous to the relationship between steady state shear viscosity $(\eta)$ and shear rate $(\dot{\gamma})$. Therefore, shear viscosities for $\dot{\gamma}=1 \mathrm{~s}^{-1}, \dot{\gamma}=5 \mathrm{~s}^{-1}$ and $7 \mathrm{~s}^{-1}$ (specific shear rates of interest for the EOR application) are compared (see Supporting Information, Table S3). This direct comparison shows that the viscosity behaviour of the new terpolymers is generally comparable to the reference copolymer, and hence the designed AMPS/AAm/AAc terpolymers remain viable.

Viscoelastic properties were also measured during frequency sweep tests. G', the elastic modulus, provides information about the reversibly stored energy in the system. G', the viscous modulus, represents the irreversible energy loss. In general, polymer solutions for EOR with higher G' and G' values (compared to a standard reference material) offer superior viscoelasticity. This is relevant for EOR, since the viscoelastic behaviour improves the sweep efficiency of the EOR process. The crossover point (that is, the frequency at which the behaviour shifts from predominantly viscous to predominantly elastic) was generally observed at very low frequencies and was occasionally not observed (especially for aqueous solution trials). This value, though commonly used as a measure of viscoelasticity, was not always observed at these low frequencies (especially since it was very early in the experimental run), and is therefore only used in a relative way as an indicator of potential EOR performance. Measurements of G' and G', for both the water and buffer solutions are shown in Figure 9 and Figure 10. 
(a)

(b)
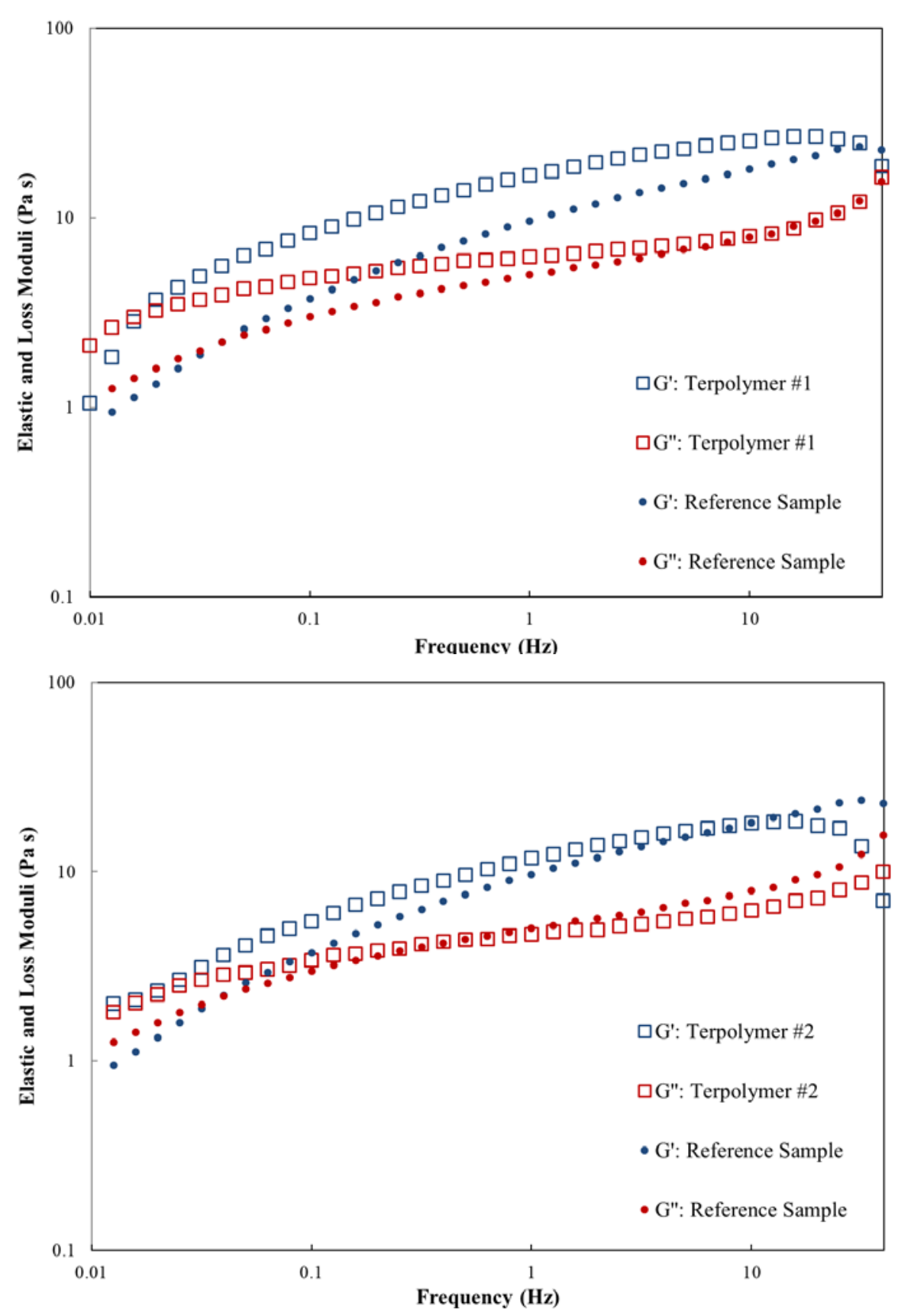

Figure 9: Elastic and Loss Modulus for AMPS/AAm/AAc Terpolymers in Water for

(a) Terpolymer \#1 and (b) Terpolymer \#2 
(a)

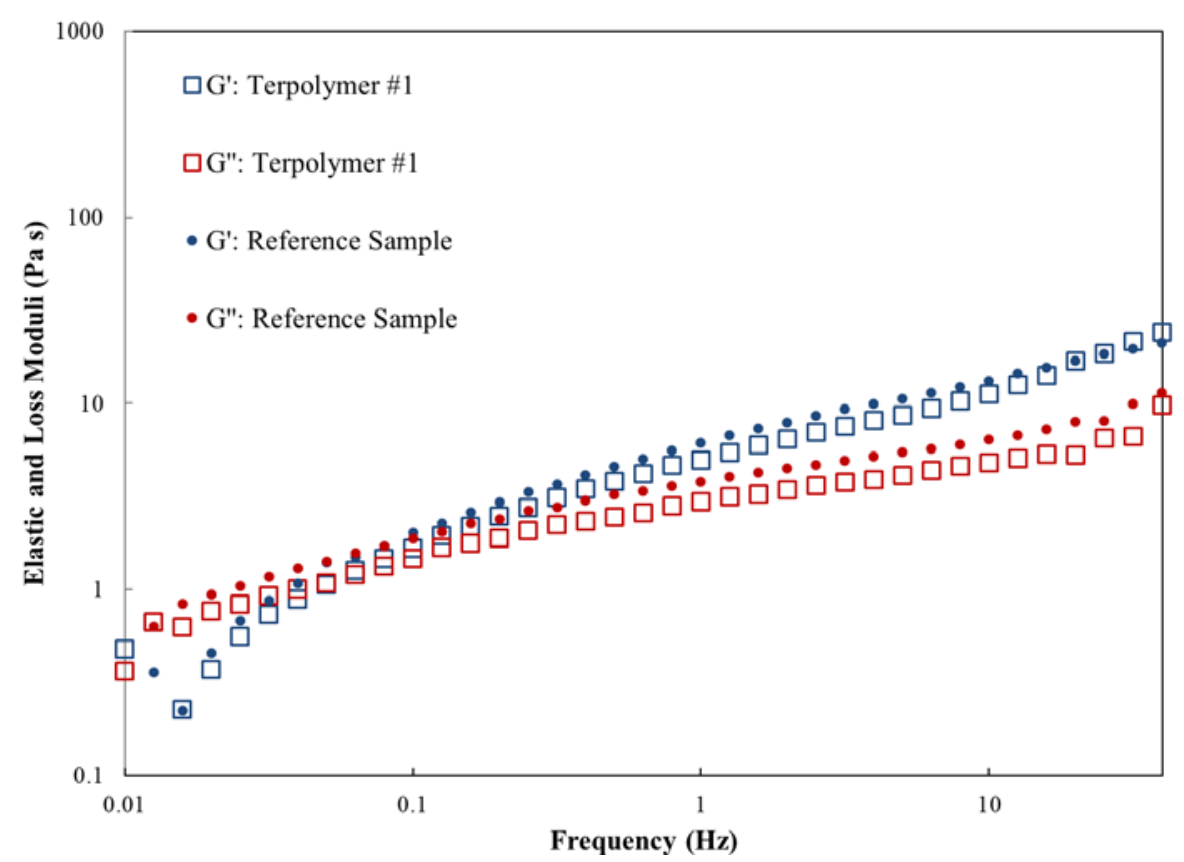

(b)

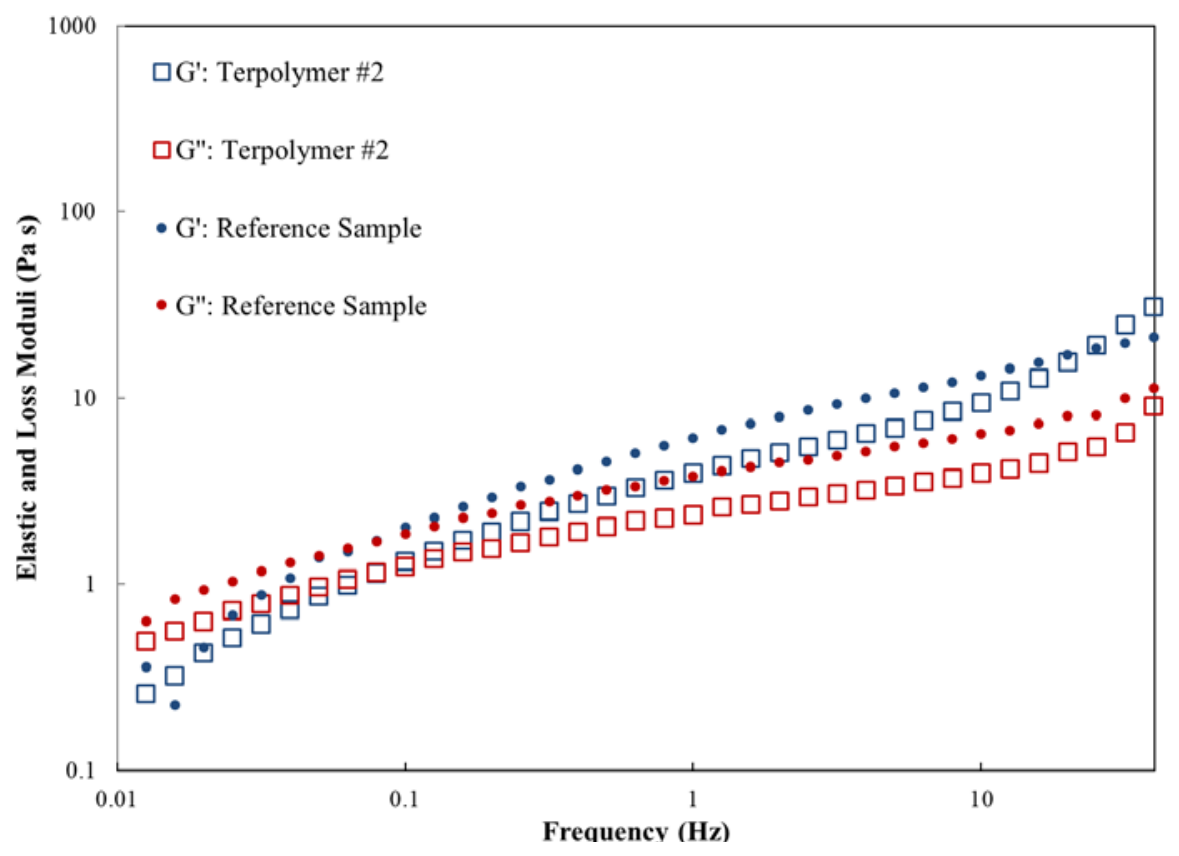

Figure 10: Elastic and Loss Modulus for AMPS/AAm/AAc Terpolymers in Buffer for

(a) Terpolymer \#1 and (b) Terpolymer \#2

As demonstrated in Figure 9, G' is generally larger than G', (except at very low frequencies). This indicates that the loss modulus dominates at very low frequencies, but that under normal 
operating conditions (that is, frequencies characteristic of the EOR application), the solutions are predominantly elastic in nature. A clear crossover point was observed for the reference sample, but the crossover behaviour occurred at very low frequencies (or not at all) for the optimal terpolymers. This predominantly elastic behaviour is desirable for polymer flooding; studies have shown that polymer solutions with higher elasticity also provide higher oil recovery efficiency [17]. In comparing the newly synthesized (optimal) terpolymers to the reference sample, terpolymer \#1 (Figure 9a) seems to be somewhat more elastic than the reference material (the moduli are higher, and the crossover point occurs at a lower frequency). However, terpolymer \#2 (Figure 9b) has characteristics that are very similar to the reference material.

Figure 10 shows the viscoelastic behaviour of the same polymeric materials in buffer solution. The properties of the newly synthesized terpolymers are comparable to the reference polymer, but the modulus values are slightly higher for the reference polymer than they are for the optimal terpolymers. For all three materials (terpolymer \#1, terpolymer \#2 and the reference material) in buffer, the elastic behaviour still dominates, but to a lesser extent than in aqueous polymer solutions. The crossover frequency in buffer is consistently higher than the crossover frequency in water, which suggests that the polymer solution behaviour is more viscous (less elastic) for a wider range of low frequencies.

We can also combine information about the storage and loss moduli by looking at the dynamic mechanical loss tangent $(\tan \delta)$. When $\tan \delta\left(G^{\prime \prime} / G^{\prime}\right)$ is below unity, elastic behaviour dominates. Low values of $\tan \delta$ (that is, high elasticity) can encourage 'pulling' behaviour in an oil reservoir, 
which assists with the removal of residual oil and increases the displacement efficiency in EOR [18].

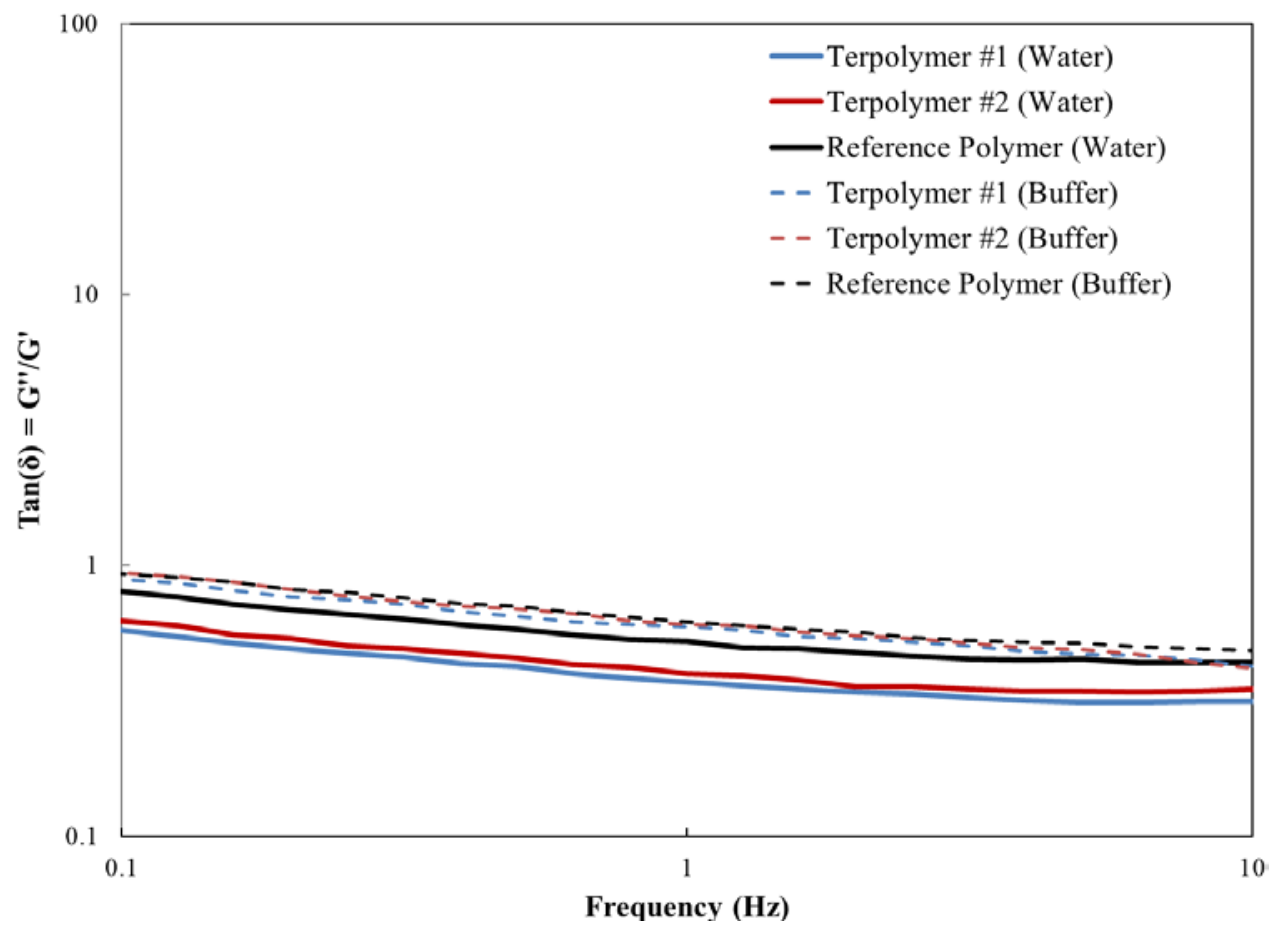

Figure 11: Dynamic Mechanical Loss Tangent (tan $\delta)$ for Designed Terpolymers and Reference Polymer

As shown in Figure 11, terpolymer \#1 and terpolymer \#2 have similar tan $\delta$ profiles. In aqueous solutions, both of the optimally designed terpolymers have a lower tan $\delta$ (therefore higher elasticity and potentially improved EOR performance) compared to the reference polymer. For the reference polymer, $\tan \delta$ values are closer to the buffer behaviour of the other materials. This indicates that the reference polymer is less affected by salts in solution, and this agrees with the complex viscosity results observed previously (Figure 8). In any case, all tan $\delta$ values (over the frequency 
range presented herein) are below unity, which adds to the increasing list of desirable properties that these terpolymers possess for the eventual EOR testing and application.

\section{Concluding Remarks}

Two optimally designed terpolymers were selected based on the results of a recent study [4] examining $\mathrm{pH}$, ionic strength and monomer concentration effects on the terpolymerization of AMPS/AAm/AAc. The formulations were selected so that the resulting AMPS/AAm/AAc terpolymers would have high molecular weight averages (on the order of $10^{6} \mathrm{~g} / \mathrm{mol}$ ), high AAm content, and a desirable microstructure (with anions well-distributed along the backbone). Given our improved understanding of polymerization recipe and operating factors on the polymerization kinetics and resulting terpolymer characteristics, the two optimally designed terpolymers were synthesized at $\mathrm{pH} 7$, ionic strength $=0.9 \mathrm{M}$, and monomer concentration $=1.5 \mathrm{M}$; the feed compositions selected were $f_{\mathrm{AMPS}, 0} / f_{\mathrm{AAm}, 0} / f_{\mathrm{AAc}, 0}=0.21 / 0.69 / 0.10$ and 0.10/0.75/0.15.

Thorough characterization confirmed that the terpolymer properties were as expected and were well-aligned with the properties of a commercially available reference material. Several independently replicated experiments allowed us to confirm that synthesis replicates and characterization replicates showed excellent reproducibility/repeatability. This is hardly ever done in the polymerization literature. We found that the cumulative terpolymer compositions were as predicted from ternary reactivity ratio estimates and that molecular weight averages were of the expected order of magnitude. Additionally, the thermal stability was improved with the incorporation of AMPS, as hypothesized. Rheological properties of the newly designed terpolymers were evaluated in aqueous solution and in buffer, and behaviours were similar to the 
reference material. We observed that the aqueous terpolymer solutions had higher shear viscosities than the aqueous reference material solution, but the reference material was less affected by the presence of salt. Also, both terpolymers had lower $\tan \delta$ profiles than the reference material, indicating higher elasticity (which often translates to improved EOR performance). Thus, investigation of several unique polymer properties confirmed the validity of our designed formulations.

At this point, we have acquired a wealth of information about the AMPS/AAm/AAc terpolymer, our model predictions thus far have been accurate, and our hypotheses for further EOR performance tests seem valid. In the future, sand-pack flooding experiments will be conducted to mimic the performance of each polymeric material in an oil reservoir. Given the promising results observed in the current study, both optimally designed terpolymers show significant potential for the enhanced oil recovery application.

\section{Supporting Information}

Experimental values and statistical analyses for molecular weight analysis (reproducibility and repeatability study) are available in Table S1 for Terpolymer \#1 and in Table S2 for Terpolymer \#2. Similarly, a summary of shear viscosities at relevant shear rates is available (Table S3). The Supporting Information is available free of charge on the ACS Publications website: http://pubs.acs.org/. 


\section{Acknowledgements}

The authors wish to thank N. Francis for her assistance with preliminary experimental work. Thanks also to J. Venne (Department of Chemistry, University of Waterloo) and R. Dickhout (Department of Chemical Engineering, University of Waterloo) for sharing their characterization expertise with NMR and TGA equipment, respectively. Additionally, the authors wish to acknowledge financial support from the Natural Sciences and Engineering Research Council (NSERC) of Canada and the Canada Research (CRC) program (AP). Special thanks to UWW/OMNOVA Solutions, USA, for special support to AJS. 


\section{References}

[1] Zaitoun, A.; Makakou, P.; Blin, N.; Al-Maamari, R.; Al-Hashmi, A.; Abdel-Goad, M.; Al-Sharji, H. Shear Stability of EOR Polymers. Proceedings of the Society of Petroleum Engineers International Symposium, The Woodlands, Texas; 2011.

[2] Sabhapondit, A.; Borthakur, A.; Haque, I. Water Soluble Acrylamidomethyl Propane Sulfonate (AMPS) Copolymer as an Enhanced Oil Recovery Chemical. Energy Fuels. 2003, 17, 683-688.

[3] Scott, A.J.; Kazemi, N.; Penlidis, A. AMPS/AAm/AAc Terpolymerization: Experimental Verification of the EVM Framework for Ternary Reactivity Ratio Estimation. Processes. 2017, 5, 9.

[4] Scott, A.J.; Duever, T.A.; Penlidis, A. The Role of $\mathrm{pH}$, Ionic Strength and Monomer Concentration on the Terpolymerization of 2-acrylamido-2-methylpropane Sulfonic Acid, Acrylamide and Acrylic Acid. Polymer. 2019, 177, 214-230.

[5] Riahinezhad, M.; Kazemi, N.; McManus, N.T.; Penlidis, A. Effect of Ionic Strength on the Reactivity Ratios of Acrylamide/Acrylic Acid (Sodium Acrylate) Copolymerization. J. Appl. Polym. Sci. 2014, 131, 40949.

[6] Mendichi, R.; Schieroni, A. Fractionation and Characterization of Ultra-High Molar Mass Hyaluronan: 2. On-line Size Exclusion Chromatography Methods. Polymer. 2002, 43, 6115-6121. 
[7] Riahinezhad, M.; McManus, N.T.; Penlidis, A. Effect of Monomer Concentration and $\mathrm{pH}$ on Reaction Kinetics and Copolymer Microstructure of Acrylamide/Acrylic Acid Copolymer. Macromol. React. Eng. 2015, 9, 100-113.

[8] Riahinezhad, M.; Romero-Zerón, L.; McManus, N.T.; Penlidis, A. Evaluating the Performance of Tailor-Made Water-Soluble Copolymers for Enhanced Oil Recovery Polymer Flooding Applications. Fuel. 2017, 203, 269-278.

[9] Randall, J.C. Monomer Distributions and Number-Average Sequence Lengths in Hydrogenated Butadiene-Styrene Copolymers from Carbon-13 Nuclear Magnetic Resonance. J. Polym. Sci.: Polym. Phys. Ed. 1977, 15, 1451-1473.

[10] Brar, A.S.; Sunita. Compositional Sequence Determination of Acrylonitrile-Butyl Acrylate Copolymers by 13C-NMR Spectroscopy. Polymer. 1993, 34, 3391-3396.

[11] Riahinezhad, M.; Romero-Zerón, L.; McManus, N.T.; Penlidis, A. Design of TailorMade Water-Soluble Copolymers for Enhanced Oil Recovery Polymer Flooding Applications. Macromol. React. Eng. 2017, 11, 1600020.

[12] Sabhapondit, A.; Borthakur, A.; Haque, I. Characterization of Acrylamide Polymers for Enhanced Oil Recovery. J. Appl. Polym. Sci. 2003, 87, 1869-1878.

[13] Zhong, C.; Luo, P.; Ye, Z.; Chen, H. Characterization and Solution Properties of a Novel Water-Soluble Terpolymer For Enhanced Oil Recovery. Polym. Bull. 2009, 62, 79-89. 
[14] El Hoshoudy, A.; Desouky, S.; Al-sabagh, A.; El-kady, M.; Betiha, M.; Mahmoud, S. Synthesis and Characterization of Polyacrylamide Crosslinked Copolymer for Enhanced Oil Recovery and Rock Wettability Alteration. Int. J. Oil Gas Coal Eng. 2015, 3, 47-59.

[15] Wu, G.; Yu, L.; Jiang, X. Synthesis and Properties of an Acrylamide-Based Polymer for Enhanced Oil Recovery: A Preliminary Study. Adv. Polym. Technol. 2018, 37, 2763-2773.

[16] Bjørkum, P.A.; Nadeau, P.H. Temperature Controlled Porosity/Permeability Reduction, Fluid Migration, and Petroleum Exploration in Sedimentary Basins. APPEA J. 1998, 38, 453-464.

[17] Wei, B.; Romero-Zerón, L.; Rodrigue, D. Oil Displacement Mechanisms of Viscoelastic Polymers in Enhanced Oil Recovery (EOR): A Review. J. Pet. Explor. Prod. Technol. 2014, 4, 113-121.

[18] Sheng, J. Modern Chemical Enhanced Oil Recovery - Theory and Practice; Gulf Professional Publishing: Burlington, USA, 2011. 


\section{Graphical Abstract}
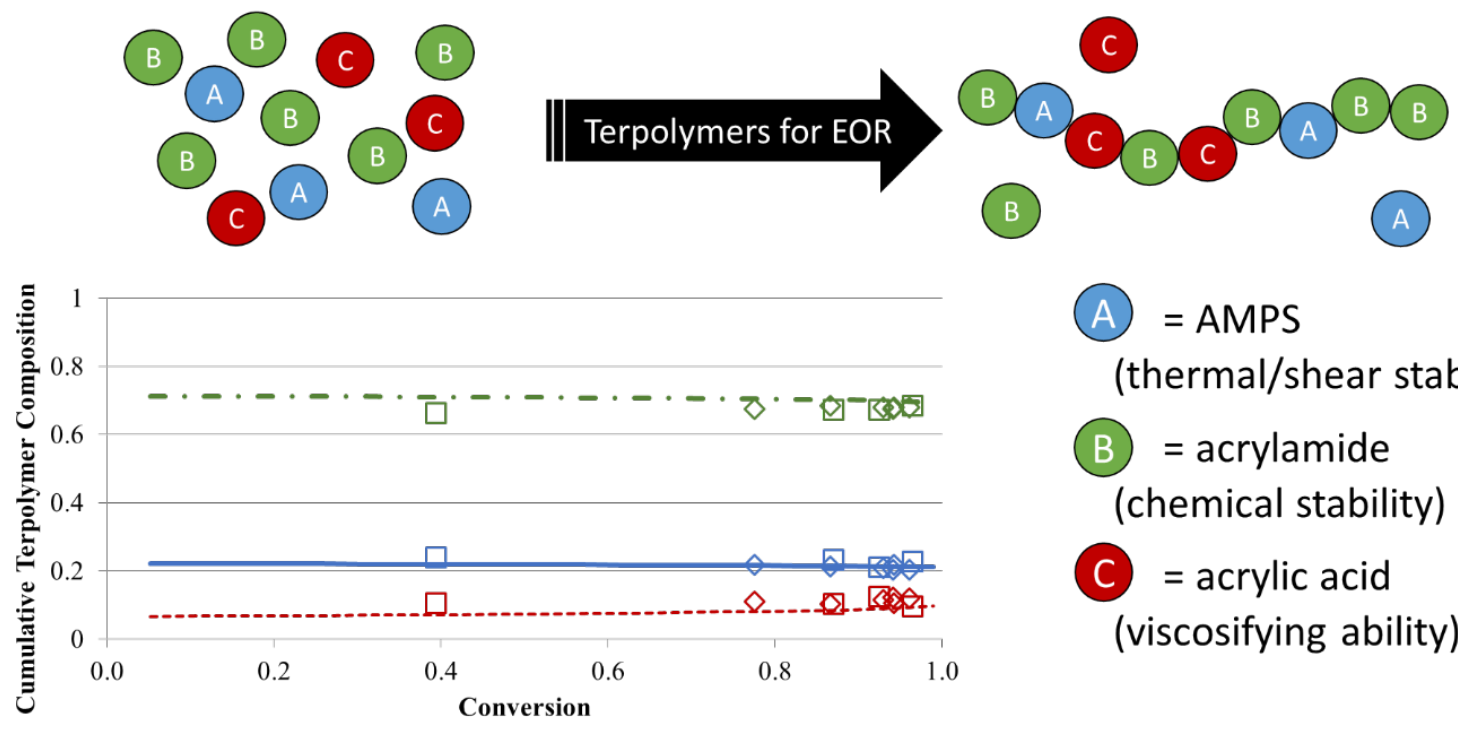

$$
\begin{aligned}
& \text { A }=\text { AMPS } \\
& \text { (thermal/shear stability) } \\
& \text { B = acrylamide } \\
& \text { (chemical stability) } \\
& C=\text { acrylic acid } \\
& \text { (viscosifying ability) }
\end{aligned}
$$

